\title{
Reasoning on Constrained Epistemic Action Modal Logic
}

\author{
Zhiling Hong \\ Software School, Xiamen University, Xiamen 361005 \\ Fujian, P. R. China \\ hongzl@xmu.edu.cn
}

\begin{abstract}
This paper put forward constrained epistemic action model logic (EAML), which extended the action modal logic and reason epistemic default logic in the dynamic epistemic theory framework. In this paper, the constrained epistemic default logic and action model logic was combined to model the dynamic cognitive update process by introducing a cognitive operator. As constrained epistemic default logic restricts the current possible epistemic state and action model logic can describe epistemic actions, the new proposed logic can better portray the cognitive operations and the restriction of the corresponding accessibility relations. The description of the logic framework was given in this study, and the related theorems were proved to show the interpretation of the constrained epistemic action model logic.
\end{abstract}

Keywords: Epistemic action model logic, Extension, Epistemic state, Multi-agent, Information

\section{Introduction}

Information is communicated, so knowledge and belief are by no means static. Dynamic epistemic logic is an umbrella term for a number of extensions of epistemic logic with dynamic operators that enable us to formalize reasoning about information change. In artificial intelligence, it is well accepted that a serious theory of planning takes into account what is known by the executing agent. In Moore's work [1], a formalism that combined aspects of both epistemic logic and dynamic logic is used to capture what needs to be known in order to carry out a plan. This work introduced the notion of epistemic precondition of an 'action', which refers to the knowledge that is needed to successfully perform it.

Based on this, H.P. van Ditmarsch, w. van der Hoek and B.P. Kooi introduced the 'action mode' approach to describe epistemic action, and then developed Action Model theory and Public Announcement Logic theory [2]. As H.P. van Ditmarsch, w. van der Hoek, and B.P. Kooi's action model logic language was defined as "cognitive operation", which expressed the cognitive operation as a cognitive modal operator. In order to complete the cognitive operation handling knowledge updating, together with express and deal with information change due to the action as a "cognitive operation", it is important to construct this dynamic recognition in the form of a language. Since "Cognitive operation" modal logic processing is through the action for the other action, the formalization of "cognitive operation" logic is an extension of the original cognitive logic theory. Obviously most of cognitive state could not be fully described by an independent modal operator and a single cognition.

Default logic represents a simple but a powerful class of non-monotonic formalisms which is one of the most prominent approaches in Artificial Intelligence for its ability to treat various forms of commonsense reasoning [3]. Reiter's default logic [4] is among the best known and most widely studied logical frameworks for reasoning from incomplete information, but Reiter's default logic was unable to reason by epistemic states. Thus, it is 
useful to give cognitive operation and develop a formalized description model, which make its belief knowledge update between the multi-agent have stronger characterization ability.

In this study, a formalized description model was given to describe the compound cognitive operation, which has stronger characterization ability to depict the belief knowledge updating between the agents in a multi-agent system. In this proposed system, the constrained epistemic default logic and action model logic was combined to model the dynamic cognitive update logic system by introducing a complex cognitive operator. Since epistemic logic deals with the current possible information and action model logic can restrict the agent's inference behavior, which make it can better portray the "translucent type" cognitive operations and the restriction of the corresponding accessibility relations.

This paper is organized as follows: in the 2 nd section, we make an introduction of action model theory and constrained epistemic default theory and then introduce the constrained epistemic action model theory $(E A M L)$, in the 3rd section, the properties and theorems of constrained $E A M L$ was proved, and in the 4th also the last section we make a summary of the whole paper.

\section{Logical Framework of EAML}

Definition 1. Let $L_{M_{U}}$ be any logical language for given parameters agent $N$, and atoms $P$, action model $(A M L)$ is a triple $M_{\vartheta}=\left\langle S, R^{a}\right.$, pre $\rangle$ such that:

1) $S \neq \phi, S$ is a domain of action operations.

2) $R^{a}: N{ }^{\circledR} 2^{S+S}$, indicates relation on $S$ for each action operation.

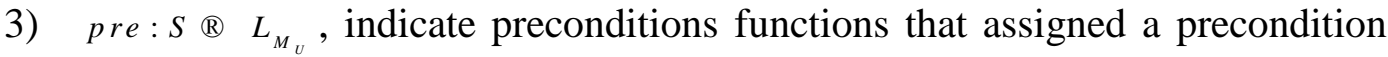
operator in $L_{M_{U}}$.

Definition 2. Let $L_{M_{U}}$ be any logical language for given parameters agent $N$, and atoms $P$, and an epistemic modal logic $\operatorname{model}(E M L)$ is a triple defined as $M_{u}=\left\langle W_{u}, R_{u}, V_{u}\right\rangle$, such that:

1) $W_{u} \neq \phi, W_{u}$ is a domain of epistemic state.

2) $R_{u}: N \rightarrow 2^{W \square W}$, or each agent $i, i \in N, R_{u}$ is a binary relation on $W_{u}$.

3) $V_{u}: P \rightarrow 2^{w}$, for each atom $p \in P, V_{u}$ is valuation on each epistemic state $W_{u}$ in $L_{M_{U}}$.

The structure $(M, w)$ indicates a basic epistemic state.

Definition 3. Give an epistemic modal logic model $(E M L) M_{u}=\left\langle W_{u}, R_{u}, V_{u}\right\rangle$ and an action model $(A M L) M_{\vartheta}=\left\langle S, R^{a}\right.$, pre $\rangle, \vartheta \in L_{M_{U}}$, for any formulas $\varphi$, if $\varphi \in L_{M_{U}}$ was truth definition in $M_{u}$ then it indicated as $\left(M_{u}, w\right) \mid=\varphi$, the semantics of formulas and actions was defined as follows:

$$
\begin{aligned}
& \left(M_{u}, w\right) \mid=p \text { iff } w \in V(p) ; \\
& \left(M_{u}, w\right)\left|=\neg \varphi \operatorname{iff}\left(M_{u}, w\right)\right| \neq \varphi ; \\
& \left(M_{u}, w\right)\left|=\varphi \wedge \psi \operatorname{iff}\left(M_{u}, w\right)\right|=\varphi \text { and }\left(M_{u}, w\right) \mid=\psi ; \\
& \left(M_{u}, w\right) \mid=K_{i} \varphi \text { iff for all } v, v \in M_{u}, \operatorname{if}(w, v) \in R_{i}, \operatorname{then}\left(M_{u}, v\right) \mid=\varphi ;
\end{aligned}
$$


$\left(M_{u}, w\right) \mid=[a] j$ iff for all $M^{\prime}, v, \operatorname{if}\left(M_{u}, w\right) \S a \cdot\left(M^{\prime}, v\right)$ then $\left(M^{\prime}, v\right) \mid=j ;$

Where $a$ is an action and $j$ a formula. The interpretation of dynamic modal formula form $[a] j$ indicates the factual epistemic states that result from executing an action $a$ and $j$ is true. §a "was defined as the subset of the domain of an epistemic operators.

Definition 4. A constrained epistemic default logic is $\langle D, W, C\rangle$, where $W$ is a set of consistent formulas of factual epistemic states, $C$ is constrained sets, and $D$ is a set of default formulas to make $W$ complete.

Definition 4. Give an epistemic modal logic model $(E M L) M_{u}=\left\langle W_{u}, R_{u}, V_{u}\right\rangle$ and an action $\operatorname{model}(A M L) M_{\vartheta}=\left\langle S, R^{a}\right.$, pre $\rangle$ on $L_{M_{V}}$, Let $M_{\Delta}=\langle D, W, C\rangle$ be a constrained $E A M L$ theory on $L_{M_{U}}$, where $W=W_{u} \cup S$, and $W$ consist of the formulas with form $[a] j$. By definition, for any formula $\operatorname{set}(S, V)$ of $L_{M_{U}}, \Gamma_{1}(S, V)$ and $\Gamma_{2}(S, V)$ are the smallest sets such that:

(1) $W \subseteq \Gamma_{1}(S, V)$;

(2) $C \subseteq \Gamma_{2}(S, V)$;

(3) $\operatorname{Th}\left(\Gamma_{1}(S, V)\right)=\Gamma_{1}(S, V)$;

(4) If $[\alpha] \varphi \in D,[\alpha] \varphi \in \Gamma_{1}(S, V)$ and $S \cup V \cup[\alpha] \varphi$ is consistent, then $\varphi \in \Gamma_{1}(S, V),[\alpha] \varphi \subseteq \Gamma_{2}(S, V)$. A set $E \subseteq L_{M_{U}}$ is a constrained extension of $M_{\Delta}$ on $F$ if and only if $E=\Gamma_{1}(E, F)$ and $F=\Gamma_{2}(E, F)$, where $F$ is called a following supplement set of $E$.

Let interpretation model $M$ be a model of formula sets $\Gamma$,if $\mathrm{f} M$ satisfies every formula in $\Gamma$.

Therefore, in a constrained $E A M L$ on $L_{M_{v}}$, the epistemic modal logic model $M_{u}$ and the action model $M_{g}$ satisfies every formula in $\Gamma$.

\section{Theorems of Constrained EAML}

Theorem 1.Let $M_{\Delta}=\langle D, W, C\rangle$ be a constrained EAML theory, then $\mathrm{E}$ is an xtension of ${ }^{M_{\Delta}}$ on $\mathrm{F}$ if and only if $E=\bigcup_{j=0}^{\infty} E_{j}, F=\bigcup_{j=0}^{\infty} F_{j}$, where $E_{0}=W, F_{0}=C$, and for $j \geq 0$ :

$E_{j+1}=\operatorname{Th}\left(E_{j}\right) \cup\left\{[\alpha] \varphi \in D, K \alpha \in E_{j}, E \cup F \cup[\alpha] \varphi\right.$ is consistent $\}$.

$F_{j+1}=F_{j} \cup\left\{[\alpha] \varphi \in D, K \alpha \in E_{j}, E \cup F \cup[\alpha] \varphi\right.$ is consistent $\}$.

Proof. According to the definitions above, we have:

(1) $W \subseteq \bigcup^{\infty} E_{j}, \quad C \subseteq \bigcup_{j}^{\infty} F_{j}$

(2) $\operatorname{Th}\left(\bigcup_{j=0}^{\infty} E_{j}\right)=\bigcup_{j=0}^{\infty} E_{j}$

(3) If $[\alpha] \varphi \in D,[\alpha] \varphi \in \bigcup_{j=0}^{\infty} E_{j}$ and $E \cup F \cup[\alpha] \varphi$ is consistent, then $\varphi \in \bigcup_{j=0}^{\infty} E_{j},[\alpha] \varphi \subseteq \bigcup_{j=0}^{\infty} F_{j}$.

Therefore by the semantics this is equivalent to 
$\Gamma_{1}(E, F) \subseteq \bigcup_{j=0}^{\infty} E_{j}, \Gamma_{2}(E, F) \subseteq \bigcup_{j=0}^{\infty} F_{j}$.

The proof of Necessity is given as follows:

Let $\mathrm{E}$ be the extension of ${ }^{M}{ }_{\Delta}$ on $\mathrm{F}$, in the following we have to prove $E=\bigcup_{j=0}^{\infty} E_{j}, \quad F=\bigcup_{j=0}^{\infty} F_{j}$.

Because $\Gamma_{1}(E, F) \subseteq \bigcup_{j=0}^{\infty} E_{j}, \quad \Gamma_{2}(E, F) \subseteq \bigcup_{j=0}^{\infty} F_{j}$, we only need to guarantee $\bigcup_{j=0}^{\infty} E_{j} \subseteq E$, $\bigcup_{j=0}^{\infty} F_{j} \subseteq F$

Following we testify that for any $j \geq 0$, we have $E_{j} \subseteq E, F_{j} \subseteq F$, therefore $\bigcup_{j=0}^{\infty} E_{j} \subseteq E, \bigcup_{j=0}^{\infty} F_{j} \subseteq F$

(1) Obviously as $E=\Gamma_{1}(E, F), \quad F=\Gamma_{2}(E, F)$, then $E_{0} \subseteq E, \quad F_{0} \subseteq F$.

(2) Suppose $E_{j} \subseteq E, \quad F_{j} \subseteq F$ and $\theta \in E_{j+1}$, in order to prove $E_{j+1} \subseteq E$, we only need to verify $\theta \in E$.

If $\theta \in \operatorname{Th}\left(E_{j}\right)$, since $E_{j} \subseteq E$, then we have $\theta \in T h(E)=E, F_{j+1}=F_{j} \subseteq F$.

If $[\alpha] \varphi \in D$, where $[\alpha] \varphi \in E_{j}, E \cup F \cup[\alpha] \varphi$ is consistent, such that $\{\theta\}$ is $j$. Because $E_{j} \subseteq E,[\alpha] \varphi \in E=\Gamma_{1}(E, F)$ holds, then according to Definition $4, \theta \in \Gamma_{1}(E, F)=E$,

$F_{j+1}=F_{j} \cup[\alpha] \varphi \subseteq \Gamma_{2}(E, F)=F$. Consequently that $\bigcup_{j=0}^{\infty} E_{j} \subseteq E, \quad \bigcup_{j=0}^{\infty} F_{j} \subseteq F$.

Since $E=\Gamma_{1}(E, F) \subseteq \bigcup_{j=0}^{\infty} E_{j}, F=\Gamma_{2}(E, F) \subseteq \bigcup_{j=0}^{\infty} F_{j}$, then $E=\bigcup_{j=0}^{\infty} E_{j}, \quad F=\bigcup_{j=0}^{\infty} F_{j}$ holds.

The proof of Sufficiency is given as follows:

Since $E=\bigcup_{j=0}^{\infty} E_{j}, \quad F=\bigcup_{j=0}^{\infty} F_{j}$, now we prove $E$ is the extension of $M_{\Delta}$ on $F$.

As $\quad \Gamma_{1}(E, F) \subseteq \bigcup_{j=0}^{\infty} E_{j}, \quad \Gamma_{2}(E, F) \subseteq \bigcup_{j=0}^{\infty} F_{j}, \quad$ then we only need to prove $\bigcup_{j=0}^{\infty} E_{j} \subseteq \Gamma_{1}(E, F), \bigcup_{j=0}^{\infty} F_{j} \subseteq \Gamma_{2}(E, F)$. Following we prove that for any $j \geq 0$, we have $E_{j} \subseteq \Gamma_{1}(E, F), \quad F_{j} \subseteq \Gamma_{2}(E, F)$.

(1)Obviously we have $E_{0}=W \subseteq \Gamma_{1}(E, F), F_{0}=C \subseteq \Gamma_{2}(E, F)$.

(2)Suppose $E_{j} \subseteq \Gamma_{1}(E, F), \quad F_{j} \subseteq \Gamma_{2}(E, F)$ and $\theta \in E_{j+1}$, in order to prove $E_{j+1} \subseteq \Gamma_{1}(E, F)$, we only need to verify $\theta \in \Gamma_{1}(E, F)$.

If $\theta \in T h\left(E_{j}\right)$ and since $E_{j} \subseteq \Gamma_{1}(E, F)$, we have $\theta \in T h\left(\Gamma_{1}(E, F)\right)=\Gamma_{1}(E, F)$, $F_{j+1}=F_{j} \subseteq \Gamma_{2}(E, F) ;$

If $[\alpha] \varphi \in D$, where $K \alpha \in E_{j}$ and $E \cup F \cup[\alpha] \varphi$ is consistent, such that $\theta$ is $j$. 
As $E_{j} \subseteq \Gamma_{1}(E, F)$, then $[\alpha] \varphi \in \Gamma_{1}(E, F)$.

And by Definition 2 we have $\theta \in \Gamma_{1}(E, F), F_{j+1}=F_{j} \cup[\alpha] \varphi \subseteq \Gamma_{2}(E, F)$, then we have $E=\bigcup_{j=0}^{\infty} E_{j} \subseteq \Gamma_{1}(E, F), F=\bigcup_{j=0}^{\infty} F_{j} \subseteq \Gamma_{2}(E, F)$ holds.

Since $\quad \Gamma_{1}(E, F) \subseteq \bigcup_{j=0}^{\infty} E_{j}=E, \quad \Gamma_{2}(E, F) \subseteq \bigcup_{j=0}^{\infty} F_{j}=F \quad$, we have $\Gamma_{1}(E, F)=E$ and $\Gamma_{2}(E, F)=F$.

Therefore $E$ is the extension of $M_{\Delta}$ on $F$.

Theorem 2. Let $M_{\Delta}=\langle D, W, C\rangle$ be a constrained EAML theory, let $E$ and $E^{\prime}$ be extensions of $M_{4}$ on $F$ and $F^{\prime}$ respectively, and $E^{\prime} \subseteq E, F^{\prime} \subseteq F$, such that $E=E^{\prime}$, $F=F^{\prime}$.

Proof. By Theorem 1 we have: $E=\bigcup_{i=0}^{\infty} E_{i}, \quad F=\bigcup_{i=0}^{\infty} F_{i} ; E^{\prime}=\bigcup_{i=0}^{\infty} E_{i}^{\prime}, F^{\prime}=\bigcup_{i=0}^{\infty} F_{i}^{\prime}$; where $E_{0}=E_{0}^{\prime}=W, F_{0}=F_{0}^{\prime}=C$, and for any $i \geq 0$, we have:

$$
\begin{aligned}
& E_{i+1}=\operatorname{Th}\left(E_{i}\right) \cup\left\{[\alpha] \varphi \in E_{i}, E \cup F \cup[\alpha] \varphi \text { is consistent }\right\} \\
& F_{i+1}=F_{i} \cup\left\{[\alpha] \varphi \in E_{i}, E \cup F \cup[\alpha] \varphi \text { is consistent }\right\} \\
& E_{i+1}^{\prime}=T h\left(E_{i}^{\prime}\right) \cup\left\{[\alpha] \varphi \in E_{i}^{\prime}, E^{\prime} \cup F^{\prime} \cup[\alpha] \varphi \text { is consistent }\right\} \\
& F_{i+1}^{\prime}=F_{i}^{\prime} \cup\left\{[\alpha] \varphi \in D,[\alpha] \varphi \in E_{i}^{\prime}, E^{\prime} \cup F^{\prime} \cup[\alpha] \varphi \text { is consistent }\right\}
\end{aligned}
$$

Suppose $E$ is not equivalent to $E^{\prime}$, then there exist the smallest $i$ for $E_{i+1} \neq E_{i+1}^{\prime}$ and $E_{i}=E_{i}^{\prime}$, such that $T h\left(E_{i}\right)=T h\left(E_{i}^{\prime}\right)$. Since there may existsome epistemic state $[\alpha] \varphi \in D$ which satisfy that $[\alpha] \varphi \in E_{i}=E_{i}^{\prime}$ and $E \cup F \cup[\alpha] \varphi$ is consistent, then by our assumption we have $\varphi \in E_{i+1}$. However $\varphi \notin E_{i+1}^{\prime}$, and $E^{\prime} \cup F^{\prime} \cup[\alpha] \varphi$ is inconsistent, thenby $E^{\prime} \subseteq E, \quad F^{\prime} \subseteq F$, we have $E \cup F \cup[\alpha] \varphi$ is inconsistent, contrary, therefore $E=E^{\prime}, F=F^{\prime}$.

Theorem 3. Every constrained $E A M L$ theory has at least one extension.

Proof. For any constrained EAML theory $M_{\Delta}=\langle D, W, C\rangle$, we construct an extension of $M_{\triangle}$ as follows:

Suppose $E_{0}=W, F_{0}=C$ and for any $j \geq 0$, let $S_{j}, U_{j}$ be the maximal set that satisfied the following two principles:

(1) $E_{j} \cup S_{j}$ and $F_{j} \cup V_{j}$ is consistent.

(2)Suppose $\theta \in S_{j}$, then there exist some default rule $[\alpha] \varphi \in D$ and $\{\theta\}$ is $\varphi$, where $[\alpha] \varphi \in E_{j}$.

$$
\text { Let } E_{j+1}=T h\left(E_{j}\right) \cup S_{j}, F_{j+1}=F_{j} \cup V_{j}, E=\bigcup_{j=0}^{\infty} E_{j} \text { and } F=\bigcup_{j=0}^{\infty} F_{j} \text {. }
$$

Suppose $\Gamma_{j}=\left\{\varphi \in E_{j}, E \cup F \cup[\alpha] \varphi\right.$ is consistent $\}$,

By the induction hypothesis, and according to the consistency of $E$, there is $S_{j} \subseteq T_{j}$. Suppose $T_{j} \not \subset S_{j}$, then there must exist a formula such that $E_{j} \cup S_{j} \cup \varphi$ is inconsistent, and 
by the semantics is equivalent to $\operatorname{Th}\left(E_{j}\right) \cup S_{j} \cup\{\theta\}$ is inconsistent. Because $E_{j+1} \subseteq E$, thus $E \cup\{\theta\}$ is inconsistent. Since $T h(E)=E$, we have $\neg \theta \in E$. As $\theta \in \Gamma_{j}$ was arbitrary, this is equivalent to $\theta \in E$, then contrary, thus $S_{j}=\Gamma_{j}$. Therefore, we have $E$ is an extension of $M_{4}$ on $F$.

These theorems have given the explanation of constrained epistemic action modal logic theory, which testified the restriction of the corresponding accessibility relations due to the cognitive operations.

\section{Conclusion}

This paper put forward a constrained epistemic action model logic, which extended the action modal logic and reason epistemic default logic in the dynamic epistemic theory framework. The new proposed logic can construct the dynamic recognition in the form of a language that the epistemic state could be fully described by an execution after action cognition. The framework deals with the current possible information and action model logic can restrict the agent's inference behavior, related theorems and properties was proved to confirmed that the new logic can better portray the cognitive operations in the cognition of the action modal logic system.

\section{Acknowledgment}

This work is supported by National Natural Science Foundation of China (31200769), the 2014 Program for New Century Excellent Talents in Fujian Province University.

\section{References}

[1] R. C. Moore, "Possible-worlds semantics for auto epistemic logic", In Proceedings of the Non-Monotonic Reasoning Workshop, (1984), pp. 344-354.

[2] H. V. Ditmarsch, W. V. D. H. A. B. K., "Dynamic Epistemic Logic (Synthese Library Series)", vol. 337, (2007), Springer Press.

[3] G. Antoniou, "Nonmonotonic Reasoning", Cambridge, MA: The MIT Press, (1997).

[4] R. Reiter, "A Logic for Default Reasoning", Artificial Intelligence, vol. 13, (1980), pp. 81-132.

[5] C. Calcagno, P. W. O'Hearn and H. Yang, "Local action and abstract separation logic", In Logic in Computer Science, LICS, 22nd Annual IEEE Symposium on, (2007) July, pp. 366-378.

[6] L. S. M. Baltag and S. Solecki, "The logic of common knowledge, public announcements, and private suspicions", in Technical Report SEN-R9922, CWI, (1999), Amsterdam.

[7] S. Kaile, "Constraints on extensions on a default theory", Journal of Computer Science and Technology, vol. 16, (2001), pp. 329-340.

[8] M. Olson, "The logic of collective action: public goods and the theory of groups", Harvard University Press, (2009).

[9] G. Aucher, "A combined system for update logic and belief revision", In Intelligent Agents and Multi-Agent Systems, (2005), pp. 1-17, Springer Berlin Heidelberg.

[10] S. Brass, "On the semantics of supernormal defaults", in Proceedings of IJCAI-93, Chambery, France, Morgan Kaufmann, San Mateo, CA, (1993), pp. 578-583.

[11] M. Kaminski and J. Rubin-Mosin, "Default theories over monadic languages", Theoretical Computer Science, vol. 364, no. 2, (2006), pp. 241-253. 